\title{
An Innovative Mechanism of Blockchain Technology on Joint Governance Model
}

\author{
Huawei Zhao and Ruzhi Xu \\ College of Finance, Qilu University of Technology (Shandong Academy of Sciences), Jinan, Shandong 250353, China
}

(Received 29 June 2020; Revised 06 March 2021; Accepted 09 March 2021; Published online 11 March 2021)

\begin{abstract}
Joint prevention and control is a social organization model dealing with the governance of public health and security incidents. The governance models should have the features of multiple subjects co-governing and distributed cooperating. Their purposes are to solve and improve the governance efficiency of dealing with public health and security incidents at the executive level. However, there are still many deficiencies in the current data governance and collaborative governance of joint prevention and control systems, which are mainly reflected in incomplete data collection, unimpeded data sharing, inflexible collaborative cooperation, and inadequate collaborative supervision. Therefore, a new innovative governance model is urgently needed. Blockchain technology is suitable for implementing multiparty data sharing and cooperation, and at the same time, it supports penetrating supervision and management. This paper studies the blockchain model for joint governance of public health and security incidents. It focuses on the multiagent collaborative prevention and control governance model, which provides a new opportunity for model innovation in data governance and in cooperative governance.
\end{abstract}

Key words: blockchain; joint prevention and control; data governance; collaborative governance

\section{INTRODUCTION}

In January 2020, a novel Coronavirus COVID-19 triggered a massive outbreak that quickly spread around the world. The prevention and control of the epidemic and pandemic need not only the intervention of the health sector but also the close cooperation of public health, security, transportation, media, logistics, community, and other departments. Therefore, to deal with public health and security incidents, governments in many countries have coordinated all their departments to establish a joint prevention and control mechanism and achieved certain results. It can be seen that joint prevention and control has become the most urgent and important working mechanism to deal with public health and security incidents, especially major incidents with high frequency and long duration such as pandemic, public opinion, mass incidents, and pollution.

In essence, joint prevention and control are to realize the cooperative governance among multiple subjects. For joint governance, the goal is prevention and control, the key is joint, and the foundation is information sharing and cooperative cooperation. Compared with emergency linkage, joint prevention and control focus on preventing and controlling the spread of the crisis, and it requires higher coordination and self-organization among equal subjects and among organizations at the grassroots level based on their respective positions and responsibilities. It relies on mass prevention and mass treatment in long-term planning and implementation. Therefore, joint prevention and control is a social organization model based on the public social governance system with multiple subject co-governance and distributed coordination, with the purpose of solving and improving the governance effectiveness of responding to public health and security incidents at the executive level.

Corresponding author: Huawei Zhao (e-mail: zhaohw@qlu.edu.cn).
Joint prevention and control should be implemented for practical use. However, in the process of implementation, the joint prevention and control of all kinds of public health and security events are frequently problematic. For example, the following problems occurred worldwide in dealing with COVID-19: the early prevention and control of the pandemic were delayed; patients could not be admitted due to poor coordination among various departments; the improper allocation of materials due to poor data collection and sharing led to a major crisis of confidence. Tracing these problems to their sources, it can be concluded that the joint prevention and control is lacking an effective data governance and cooperative governance model. In other words, "data governance and cooperative governance" are an important and difficult goal for joint prevention and control.

Fortunately, with the development of blockchain technology [1], many studies showed that the joint prevention and control based on blockchain was an important grasp to achieve this goal [2]. The main contribution of this paper is to further verify the significance of blockchain to address the joint prevention and control problem. The rest of the paper is organized as follows. Section II discusses the related work. Section III introduces the three major challenges for joint prevention and control. Section IV explains why blockchain is the new opportunity for joint prevention and control governance model innovation, and section $\mathrm{V}$ presents the effect mechanism of blockchain on the innovation of joint prevention and control governance model. Section VI discusses the recommendations and combination of the other cutting-edge technologies that can further advance the blockchain governance structure, section VII concludes the paper.

\section{RELATED WORK}

Joint prevention and control are an important way of responding to emergencies and have been widely used in public health incidents, 
air pollution prevention, and other emergency scenarios. To improve the efficiency of joint prevention and control, many studies have been performed in the following aspects.

\section{A. PANDEMIC PREVENTION AND CONTROL}

In the scenario of epidemic and pandemic prevention and control, to achieve a highly efficient transition between patients and medical resources, some researchers proposed to establish a comprehensive application system involving a three-dimensional transportation to optimize the match between traffic resources and medical resources, which will maximize the transportation used for connecting resources. The researchers proposed a joint prevention and control system, discussing the functions of the Internet of Things, the communication technology, and traffic control methods during the prevention and control of COVID-19 and patients' treatment [3].

In the field of cross-regional joint prevention and control, some researchers concluded major measures and experience of cross-regional collaborative prevention and control, compares the difference between domestic and overseas, and puts forwards major strategies of cross-regional collaborative prevention and control in response to major pandemics, including: improving the system and mechanism of interregional coordination and strengthening the linkage of regional emergency command [4]; strengthening the sharing of cross-regional information monitoring and using new technologies to improve the ability of rapid response; coordinating and dispatching resources across regions to fight COVID-19 and establishing a mechanism for regional mutual assistance [5]; implementing regional traffic control and strengthen fine management in key cross-border areas; taking targeted measures to prevent and control the pandemic in different regions and at different levels, and developing differentiated prevention and control measures; promoting regional enterprises to steadily resume work and production to ensure the smooth return of employees [6].

To analyze the function of big data to pandemic joint prevention and control, some researches pointed out that the advantages of big data are reflected in the following aspects:

(1) The personal information of users in the affected areas obtained by telecom operators and Internet companies can be anonymously processed and summarized for analysis, so as to form information such as the distribution of crowd gathering hotspots and the flow of people across regions, which can be used for the prediction and analysis of the development of the pandemic and the scheduling of medical resources.

(2) Conducting retrospective analysis of payment or transaction information, accommodation information and other data occurring in or near the pandemic point, conducting "contact tracing" to find persons with exposure history of the pandemic point or the pandemic area as soon as possible, conducting medical observation and isolation as soon as possible to cut off the source of infection. And using a blockchain-based consensus checking to mage big data in cloud storage to keep data collected from different locations to be consistent [7].

(3) Retrospectively analyzing the geographical location of patients, pathogen carriers, suspected patients, and other data to reconstruct the track of their movements during the time period, so as to detect and warn close contacts as soon as possible.
At the same time, to protect the information security of joint prevention and joint, these researchers proposed the following strategies [8]:

(1) Clearing the legal basis and authorized subject of big data supporting joint prevention and control work.

(2) Defining the basic principles for the collection of personal information necessary for joint prevention and control.

(3) Clearing that personal information limited to pandemic prevention and control and disease prevention and control shall not be used for other purposes, and citizens' personal information shall be desensitized when it is disclosed in accordance with the necessity of joint prevention and control work.

(4) Clearing the requirements for collecting or mastering personal information security and protection in joint prevention and control work.

In the field of coordination of joint prevention and control and emergency management, some researchers pointed out that responsibilities should be clear in each level of joint prevention and control to realize vertical coordination and coordination at the same level. To realize the coordination, five collaborative mechanisms should be established: coordination between normal mechanism and emergency mechanism, coordination between risk prevention and resolution mechanism and emergency response mechanism, coordination of operation mechanism and evaluation mechanism of emergency response, coordination of emergency monitoring mechanism and reward and accountability mechanism, coordination of emergency termination mechanism and material compensation mechanism [9].

\section{B. POLLUTION PREVENTION AND CONTROL}

In the scenario of pollution prevention and control, Suo [10] and Shu et al., [11] pointed out that establishing a system of cogovernance is the fundamental solution to regional air pollution. However, in terms of the current situation of joint prevention and control of air pollution, the crux of air pollution co-governance system lies in the blurred boundary of governance, lack of participants, and poor interactions, thus lacking an effective endogenous driving force. And then, the researcher believed that the innovation of air pollution governance mode is to realize the transformation from traditional governance system to data-driven governance system.

Chai et al. [12] believed that the mechanism of regional joint prevention and control of air pollution has some problems, such as insufficient basic research, lack of practical action, lack of corresponding policy and legal support, and lack of interdepartmental coordination. And then, they pointed out a five-goal mechanism, that is, unified planning, unified monitoring, unified supervision, unified assessment, and unified coordination.

Studies [13-15] suggested that the establishment of a longterm and effective mechanism of regional air pollution joint prevention and control is beneficial to the development of regional air environmental management. They pointed out that establishing regional atmospheric environmental management agency, implementing ecological compensation for atmospheric pollution, strengthening enforcement of laws, and regulations as well as expanding public participation are effective mechanisms to improve the air pollution joint prevention and control.

From the above studies, we can conclude that most research fruits are focus on macroscopic plans and methodology of joint prevention and control mechanisms, and there is little researches 
are developed from the view of data governance, especially data governance realized by blockchain.

\section{THE THREE MAJOR CHALLENGES FOR JOINT PREVENTION AND CONTROL}

Currently, the joint prevention and control mainly face three major challenges in the fields of data governance and cooperative governance.

\section{A. INFORMATION COLLECTION AND REPORTING CHANNELS ARE NOT SMOOTH}

The key to the prevention and control of public health and security incidents lies in early warning, and the key to early warning is to launch the joint prevention and control of grassroots institutions to carry out effective information collection and reporting. However, current joint prevention and control mechanisms cannot effectively organize institutes and people to take an active part in all kinds of information collection and report-related public health and security. It means that the superior institution cannot use full-dimension information to accurately grasp the emergence of public health and security incidents, which has seriously restricted the effective management of public health and security incidents, such as the pandemic situation, public opinion, mass incidents, and pollution.

In the early stage of COVID-19, global countries failed to realize early surveillance and early warning of COVID-19, which is a prominent manifestation of the lack of information collection and reporting channels.

\section{B. FILTERING EFFECTIVE INFORMATION IS DIFFICULT}

In the joint prevention and control, the more timely and accurate data collected and reported, the more conducive to the early warning and early disposal of public health and security incidents. However, when public health and security incidents are in their initial stage, their characteristics are often not obvious, and it is impossible to clearly understand and describe them. It then leads to the aggregation of a large number of false data in the joint prevention and control system, which makes it difficult to timely screen effective information, and ultimately leads to the failure of early warning of public health and security incidents.

In this COVID-19 outbreak, the pandemic prevention and control systems in global countries have exposed the difficulty in quickly identifying new pandemic outbreaks from the large number of cases of viral pneumonia each winter.

\section{OTHER AREAS OF IMPERFECT COORDINATION AND CO-GOVERNANCE}

Joint prevention and control focus on creating a cooperative governance of multiple subjects and should highlight "effectiveness" and "dynamic" in the word "joint." However, the current cooperative and co-governance mechanisms of joint prevention and control are not perfect. It is unable to achieve effective coordination of actions among agencies at the same level with different responsibilities, and difficult to implement flexible and dynamic selforganization coordination according to the evolution and progress of public health and security incidents, which causes joint prevention and control unable to play the maximum effectiveness.
In the process of joint prevention and control of COVID-19, many hospitals did not treat patients promptly, which highlighted the problem of ineffective cooperative governance of multiple subjects.

\section{THE NEW JOINT PREVENTION AND CONTROL GOVERNANCE MODEL}

At present, social governance shows a trend of integration and innovation with modern science and technology. Many studies pointed out that promoting blockchain and other high-tech applications plays an important role in improving the scientific and precise level of social governance. This trend fully shows that it has become a consensus to promote the modernization of social governance system and governance capacity by applying the high-tech which is represented by blockchain technology.

In the early stage of the development of blockchain, its characteristics such as data untamability, traceability, and robustness of data storage system make people tended to focus their attention on data governance. Some researchers believed that blockchain technology had the characteristics of distributed, tamperresistant, and traceable, and it could solve the problem of data island and data ownership confirmation, realize trust coordination across regions and institutions, and improve collaboration efficiency in the field of public health and security governance [16]. Other researchers considered that data sharing based on blockchain to be a big step forward in data sharing [17] and had a protection mechanism for sensitive data, which could resolve trust issues between partners and conduct institutions that did not trust each other to reach a consensus in cooperation [18].

With the continuous deepening of people's understanding of blockchain, the effect of blockchain on the innovation of multiagent collaborative governance model has attracted more and more attention. Some researchers propose that blockchain uses a decentralized, highly trusted way to jointly maintain a reliable database, and is suitable for collaborative governance of open educational resources [19]. Some researchers believe that blockchain-based token economy will be another upgrade of the human trust model and cooperation model, which will contribute to the formation of a large-scale social governance system with low-friction coefficient of trust and high collaboration efficiency [20]. Some researchers think that the technical characteristics of blockchain enable it to be widely used in governments, social governance, and organization building, so as to promote the modernization of organizational governance system and governance capacity [21].

It can be seen that researchers have gradually realized the positive role of blockchain in social governance innovation. However, the main research is still at the macro level and has not been specific enough to study an application scenario from the executive level. This paper focuses on the application of blockchain in the field of joint prevention and control, and it shows that when blockchain is used in joint prevention and control, it not only can show the advantages in data governance such as data untamability and traceability but also can show the following advantages in multiagent cooperative governance.

\section{A. SUPPORTING DIVERSE COLLABORATION PATTERNS}

The essence of the consensus mechanism of blockchain is that multiparty verify and vote on transactions that need to be stored in 
the blockchain, which is the basis for multiparty trust and collaboration. With the development of blockchain technology, the diversified consensus mechanism can realize the collaboration of different models. (1) Broadcasting model: One node broadcasts a message, and other nodes store the message after verifying the validation of the message. This model can be used for command transmission in joint prevention and control. Its advantage lies in that it can realize unified receiving and storage of commands, and avoid the denial of command by the command issuer. (2) Voting model: One party broadcasts the message, multiparty verify and vote the message, and decide whether store the message on blockchain or not according to concrete voting mechanism. This model can be used for collective decision in the process of joint prevention and control. Its advantage is that participants can share their own core data indirectly through voting behavior, without directly sharing their own core data. (3) Supervision model: This model is an upgraded version of the voting model, that is, in the voting consensus process, a node has a veto power. In the joint prevention and control, this model can realize the regulatory authorities' penetrating supervision of concrete business. Its advantage lies in that it can prevent the illegal business in real time and strengthen the risk prevention and control ability of the joint prevention and control.

\section{B. BREAKING SEPARABLE GOVERNANCE STRUCTURE}

At present, social governance is becoming more and more complicated. It is an effective means to deal with the complex social governance to construct multilayer collaborative complex network and promote the sharing of resources and data among multiple subjects and the complementation of capabilities and advantages through multilayer collaboration [22]. However, at present, social governance structure also shows obvious separable characteristics, which leads to fragmentation, extensive and inefficient social governance [23]. With the development of blockchain, the crosschain mechanism and the layered sharding technology of blockchain are conducive to breaking the separable governance structure and realizing the innovation of governance model.

Cross chain is used to realize the transfer of value and information between different blockchain systems. This technology plays an important role in breaking the information barrier and realizing the information transmission and sharing between different systems.

Layered sharding is a kind of expansion technology of blockchain. Its main idea is to improve the efficiency of blockchain transaction accounting through parallel consensus design, to improve the transaction processing capacity of the system. Sharding: The whole network node of the blockchain is divided into different sets, each set independently executes the consensus protocol to check and store relevant transactions, to achieve the effect of parallel transaction processing. Layering: as an extension of the sharding mechanism, the nodes of the blockchain are divided into layers to form a hierarchical blockchain network. The nodes of the lower layer adopt the sharding mechanism for parallel consensus, and the nodes of the upper layer are responsible for verifying and storing transactions of each shard.

The cross-chain and layered sharding mechanism enable the blockchain to build a cross-chain sharable, horizontally extensible, and vertically transmissible network architecture, which is very suitable for the multilevel joint prevention and control governance based on multiparty data sharing and cooperation.

\section{EFFECT OF BLOCKCHAIN ON INNOVATION OF JOINT PREVENTION AND CONTROL GOVERNANCE MODEL}

Joint prevention and control of public health and security incidents require good cooperation among all participants, and the prevention and control effect can be maximized through data sharing and cooperation among multiple participants. Blockchain can realize the trusted sharing of data, promote the coordination and process optimization of business, and strengthen the supervision and regulation of transactions. Its advantages in data governance and collaborative governance contribute to the data sharing and cooperation of joint prevention and control, and promote the innovation and upgrading of its governance model.

\section{A. OPTIMIZING THE INFORMATION-SHARING MECHANISM IN JOINT PREVENTION AND CONTROL}

1) EXEMPTION. The focus of joint prevention and control is at the grassroots level. Due to poor professional personnel and equipment, grassroots organizations often give up reporting suspected public health and security incidents for fear of misreporting, thus missing the early warning of public health and security incidents.

The consensus mechanism of blockchain can realize the multiparty consensus of messages. When a message is broadcast in the blockchain system, it could be stored in the blockchain only after the multiparty consensus. Thus, messages in the blockchain will have the endorsement of multiparty. This will remove the doubts of grassroots organizations when reporting suspected public health and security incidents, and help improve the ability of joint prevention and control in early warning of public health and security incidents.

For example, if a blockchain consists of four nodes, $N 1, N 2$, $N 3$, and $N 4$, as shown in Fig. 1 . When node $N 3$ wants to submit message $M$ into blockchain, it must broadcast $M$ to $N 1, N 2$, and $N 4$. If $N 1, N 2$, and $N 4$ believe $M$ is valid, they will vote $M$ to be recorded into blockchain, which means $N 1, N 2$, and $N 4$ endorse $M$, and all of them will be responsible for $M$.

2) INCENTIVE. Early warning for joint prevention and control of public health and security incidents is a long-term and persistent work. In the long process of early warning and monitoring, grassroots organizations tend to be slack and neglect the collection and early warning of public health and security incidents.

Under the blockchain architecture, institutions that actively participate in reporting can get rewards in the form of credits and tokens based on the unique incentive mechanism of blockchain. This will effectively encourage grassroots agencies of joint prevention and control to actively report suspected information of public health and security incidents, and it provide support for early

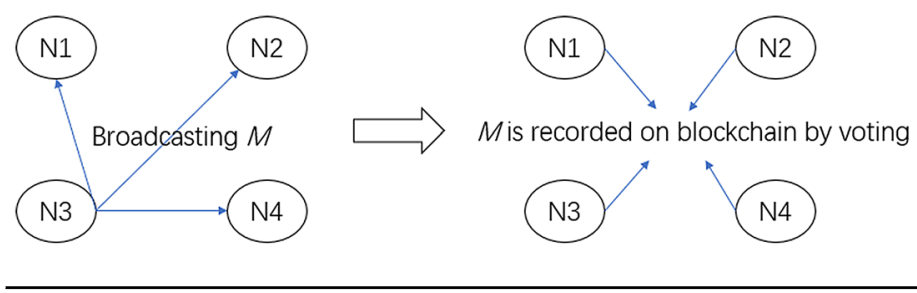

Fig. 1. Multiparty endorsement. 
warning of public health and security incidents and timely decision of decision-making institutes.

3) COMPREHENSIVE. Timely and comprehensive data are crucial to the prevention and control of public health and security incidents, especially early warning. Under the layered sharding blockchain architecture, various agencies involved in joint prevention and control can build a layered sharding blockchain network based on subordinate relations and segmented according to territorial relations. Each sharding forms a subnet responsible for local data collection. At the same time, different organizations can use cross-chain technology to realize data sharing. In this way, a huge omnidirectional data acquisition network can be built, which can collect data related to public health and security incidents from different dimensions and provide full-dimensional data support for joint prevention and control decisions.

As shown in Fig. 2, in joint prevention and control, each domain or area can build a blockchain to collect messages, all nodes in a blockchain record messages into their blockchain by consensus mechanism. In particular, an upper node, for example, a node belonging to the second-level institute, will take part in the consensus of the lower blockchain, to transfer the messages from the lower blockchain to the upper blockchain. In addition, blockchains in the same level can share data by cross-chain protocols.

Thus, the architecture in Fig. 2 can build a comprehensive message collection system.

4) EFFECTIVE. The active reporting of suspected public health and security incidents by grassroots organizations will inevitably lead to the impact of a large number of invalid data for joint prevention and control, which will seriously reduce the effectiveness of timely handling of public health and security incidents. For example, the current reporting system for disease pandemic is difficult to screen the vast number of suspected cases for valuable information about unknown outbreaks.

Under the blockchain architecture, the report of public health and security event data needs to be subject to multiparty consensus, and a large number of false data will be eliminated in the consensus stage, which will greatly improve the quality of the reported data and help the superior institutions to obtain effective data for timely processing.

5) SHARING. In the voting part of the blockchain consensus mechanism, each participating node will make voting judgment on a broadcast event based on its own data. In essence, nodes do not need to share their own original data but only need to share the judgment and decision information based on the original data. On the one hand, it can protect the original data of nodes from disclosure. On the other hand, it also gives full play to the value and role of the original data of nodes. Therefore, it can greatly enhance the willingness and motivation of participating nodes to actively share information in joint prevention and control.

\section{B. OPTIMIZING THE COOPERATION IN JOINT PREVENTION AND CONTROL}

1) TRANSFORMATION OF GOVERNANCE FUNCTIONS. Effective joint prevention and control require the governance organization to avoid the all-encompassing governance model, and to change its functions to realize the coordinated and co-governance with the participation of all kinds of social subjects under macrocontrol. Joint prevention and control under the blockchain framework can clearly define the boundaries of governance power and effectively play the regulatory role of the governance organization.

For example, under the broadcasting model of consensus mechanism, the governance organization can broadcast instructions to the blockchain network under its jurisdiction to realize the unified deployment of all participating. Under the voting model of consensus mechanism, the governance organization can vote and register the broadcast messages in the blockchain to realize the supervision of multiparty cooperation. Under the supervision model of consensus mechanism, the governance organization

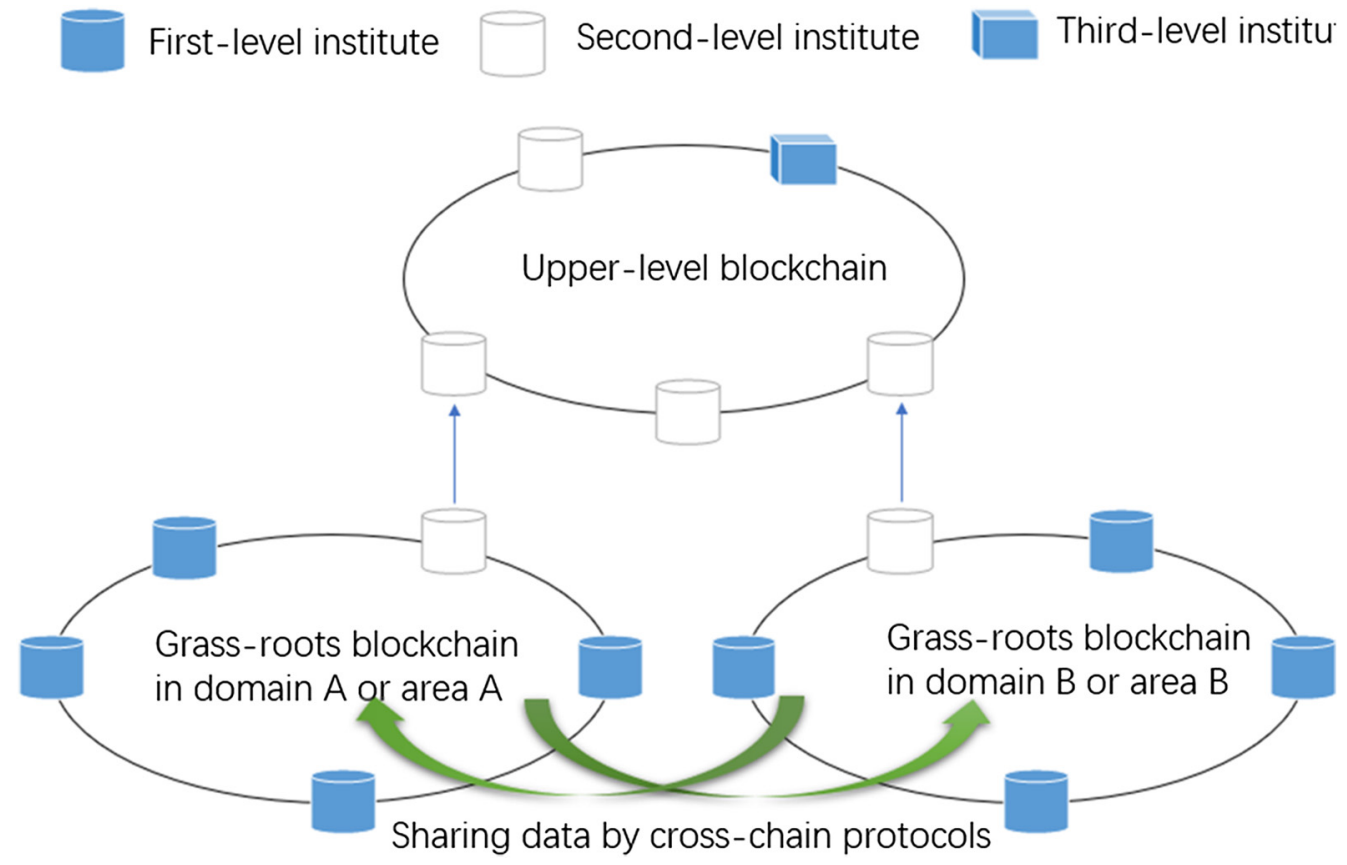

Fig. 2. Comprehensive messages collection system. 
can implement a veto to prevent violations in time and realize penetrating supervision.

2) TRUST IN MULTIPARTY COLLABORATION. In the process of joint prevention and control, effective cooperation between multiple parties must be based on mutual trust, and in this field, blockchain technology has great advantages. On the one hand, a prevention and control governance center monitoring nodes can be added to the blockchain network to audit and supervise the broadcasting behaviors of other participating nodes, which will form an effective deterrent for nodes' attempts to lie. On the other hand, data on the blockchain have features of untamability, nonrepudiation, and traceability. If a node puts false messages on the blockchain, the message will become an indelible "stain", and an evidence of punishment of the node, which makes the node's lying cost very high. Therefore, the introduction of blockchain technology is conducive to enhancing mutual trust among multiple actors in joint prevention and control and promoting trusted cooperation.

3) DYNAMIC AUTONOMY. Public health and security incidents have a dynamic evolving process, and their features will evolve with the change of time and space, and corresponding prevention and control strategies and coping methods need to be adjusted constantly. Therefore, dynamic autonomy between participants is particularly important in joint prevention and control.

Under the blockchain architecture, the parties can vote to decide the phase and the characteristics of public health and security incidents, and on this basis, these parties can dynamically vote the dominant party of joint prevention and control. It is conductive to form a dynamic and autonomous joint prevention and control architecture with multiple subcenters. Such an organization is of great value for improving the overall coordination of joint prevention and control governance, promoting the tight coupling between the functions of participants, and realizing the multiplier effect of joint prevention and control governance.

As shown in Fig. 3, in a joint prevention and control of a public health and security incident scenario, node $\mathrm{A}$ in the blockchain is the dominant node in stage 1 , and when the public health and security incident develops into stage 2, its features changes. As

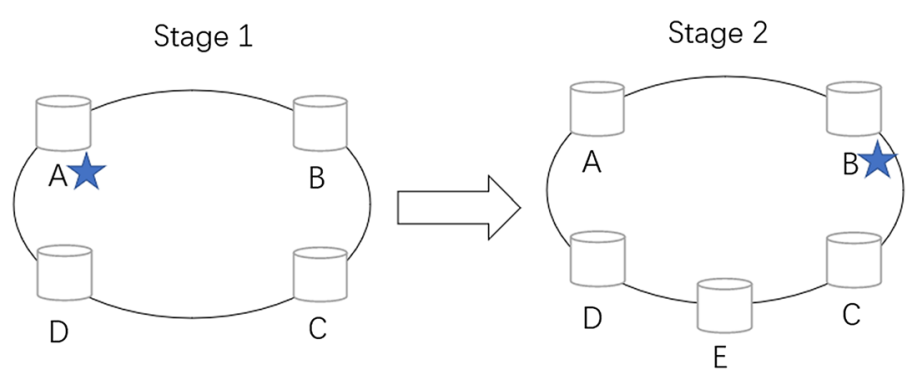

Fig. 3. Dynamic autonomy. the situation requires, the nodes in the blockchain can vote node $\mathrm{B}$ as the dominant node, and vote node $\mathrm{E}$ as a new node of the blockchain.

4) COORDINATED SUPERVISION. Effective supervising of all aspects of public health and security incidents is an important guarantee to ensure the compliance and legality of joint prevention and control. At the same time, supervision should be multisubjective and multichannel to reflect the openness and transparency.

Under the blockchain architecture, on the one hand, the governance regulatory department can participate in the operation of the blockchain as a node, and every information on the blockchain will be kept for bookkeeping by the governance node, so as to realize the penetrating supervision at the business level and ensure the real effectiveness of the supervised information. On the other hand, each information in the blockchain is recorded by each participant of the blockchain (such as news media, government departments, and functional institutions), so as to achieve a seamless group supervision and promote the governance of joint prevention and control to be more standardized.

\section{EXAMPLE ANALYSIS}

In an epidemic and pandemic joint prevention and control-systembased blockchain, multiple grass-roots health agencies and a higher health agency $R$ can construct a blockchain network $N 1$, as shown in Fig. 4. When a grassroots health agency $A$ finds a case of suspected pandemic $m$, it will broadcast $m$ in $N 1$. And when another health agency $B$ in $N 1$ finds the same case, $B$ will confirm the case. Once the majority agencies confirming the suspected case $m$ reaches a certain threshold value, $m$ will be recorded into the blockchain network $N 1$. At this time, $R$ will receive the case of suspect pandemic, and it will broadcast the case in a higher blockchain $N 2$, where $R$ as a member.

In the mechanism, $A$ is willing to broadcast $m$ to $N 1$, and there are two reasons. The first one is that once $m$ is submitted to N2, it means that $m$ is confirmed by majority agencies in N1. At this situation, $m$ will have multiparty endorsement, and $A$ does not take responsibility alone. The second one is that once the submitted $m$ is the true case of pandemic, $A$ will receive an award from blockchain. For the same reasons, other agencies in $N 1$ are willing to confirm $m$ when they believe $m$ is a suspected case of pandemic.

In addition, because $R$ is a higher health agency and has more information, $R$ can be given the veto power. When $R$ knows that $m$ is a false case of pandemic, it can stop recording $m$ into $N 1$, and stop $m$ from spreading it to $N 2$. Thus, $R$ can realize penetrating supervision to the lower agencies and stop the spreading of the false messages.

To achieve the better-coordinated supervision, other related institutes can take part in $N 1$ as members and supervise the operation of the epidemic and pandemic joint prevention and control system, which will achieve a seamless group supervision.

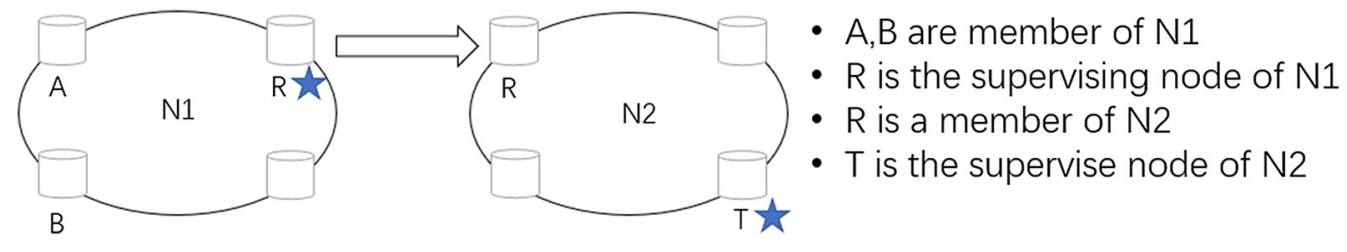

Fig. 4. An example of joint prevention and control-system-based blockchain. 
Once the pandemic outbreaks, the function of the epidemic and pandemic joint prevention and control system will turn from the prevention to taking measures. In this stage, $R$ will turn its role from supervision to organization, and it will broadcast many orders to grassroots health agencies in $N 1$ to against the pandemic. Thus, the joint prevention and control-system-based blockchain realizes the dynamic autonomy according to the development of pandemic.

\section{RECOMMENDATIONS}

Through data governance and collaborative governance, blockchain technology can solve the problem of difficult to share data and collaborate caused by separable governance structure, which is conducive to forming the resultant force of multiagent governance, promoting the maximization of the utility of resource allocation, and realizing the improvement of the effectiveness of joint prevention and control governance. Thus, the fusion of blockchain and joint prevention and control is an important innovation of the existing concept, model, path, and mechanism of joint prevention and control governance, and an important embodiment of the modernization of joint prevention and control governance. To better play the role of blockchain technology in joint prevention and control, policies should be appropriately tilted in the following aspects:

\section{A. PROMOTING OF INTEGRATION OF BLOCKCHAIN WITH OTHER INFORMATION TECHNOLOGY}

The integration of blockchain and Internet of Things (IoT) can enhance the credibility of blockchain data, where interconnected units require data integrity and trust [10]. The integration of blockchain and cloud computing can provide a common blockchain service platform and offer greater capacity for performing required computation and communication [24]. The integration of blockchain and big data can improve the density of valuable data, and the integration of blockchain and artificial intelligence can accelerate the evolution of intelligent algorithms [7].

It can be seen that the integration of blockchain and other information technologies can not only give full play to the advantages of blockchain itself but also provide stronger support for data collection, analysis, and intelligent processing of joint prevention and control.

In addition, when blockchain is integrated with other widely used information technologies, people's cognition of blockchain technology will be deepened, which will promote its application in joint prevention and control.

\section{B. BUILDING BLOCKCHAIN ON SERVICE PLATFORM}

In essence, joint prevention and control is a kind of social governance model in which various institutions are extensively involved under the regulation of the governance. Therefore, it is necessary for the governance organization to build a public blockchain service platform providing joint prevention and control public services. Comprehensive discussions and systematic implementations of such service platforms are available in [25].

On the one hand, it can avoid the waste of resources caused by repeated construction of various institutions; on the other hand, it can improve the credibility and appeal of the platform and guide all institutions to actively participate in the joint prevention and control of public health and security incidents.

\section{ACCELERATING TECHNICAL STANDARDS SYSTEM}

The joint prevention and control of public health and security incidents require extensive participation and interaction of institutions at all levels. To realize effective data governance and cooperative governance among multiple institutions, it is necessary to accelerate the development of the blockchain technical standards system. For example, the standard system should include standards of data broadcasting, standards of data storage, standards of consensus mechanism, and so on.

\section{CONCLUSIONS}

In this paper, we first analyzed the problems existing in the joint prevention and control and discussed the advantages of applying blockchain to the joint prevention and control governance systems. Then, we proposed an innovative function of data governance and cooperative governance mechanism based on blockchain to address all of the aspects in joint prevention and control systems. The study presented a new view for the joint prevention and control and offered a good guiding significance for building a new and efficient joint prevention and control governance model. The model can improve the joint prevention and control governance system and enhance the public security governance capacity of the society.

\section{ACKNOWLEDGMENT}

This work was supported by "Shandong Social Science Planning and Research Project"/“Shandong Academy of Social Sciences Innovation Project" (20BCXJ01) and Shandong Provincial Major Technology Innovation Projection under Grant 2018CXGC0703.

\section{REFERENCES}

[1] M. L. Mao and J. Y. Lu, "Research on block chain technology and its application in government governance," E-Government, vol. 2, pp. 2 14, Feb. 2018, DOI: 10.16582/j.cnki.dzzw.2018.02.001.

[2] X. X. Qi, "The application of block chain technology in government data Governance," J. Beijing Inst. Technol., vol. 3, pp. 187-194, 2019, DOI: 10.15918/j.jbitss1009-3370.2018.5150.

[3] L. Niu, Y. W. Jing, and L. Luo, "Discussion on the application of intelligent transportation in epidemic prevention and control," Shandong Sci., vol. 33, no. 2, pp. 17-21, Apr. 2020.

[4] O. Y. Peng, X. Y. Liu, and Y. C. Zhong, "Cross regional collaborative prevention and control mechanism in response to major epidemics," Planners, vol. 36, no. 5, pp. 61-66, 2020.

[5] C. H. Zhou, T. Pei, and Y. Y. Du, "Big data analysis of COVID-19 and establishment of regional prevention and control policies," Proc. Chin. Acad. Sci., vol. 2, pp. 200-203, 2020.

[6] G. J. Cheng, H. Qi, and G. F. Zuo, "Joint regional prevention and control of major diseases," J. Prevent. Med. Intell., vol. 1, pp. 90-92, 2007.

[7] G. Qi, Z. Zhu, M. Haner, J. Sim, J. Sun, Y. Chai, Y. Chen, and Y. Li, "Blockchain based consensus checking in cloud storage," in Proc. IEEE 14th Int. Symp. Auton. Decentralized Syst., Utrecht, the Netherlands, April 2019, pp. 49-56. 
[8] Y. Q. Hong, "Big data supports the analysis of personal information protection in joint prevention and control," Inf. Secur. China, vol. 2, pp. 30-32, 2020.

[9] C. G. Wang, "Coordination of emergency management from the perspective of joint prevention and control," China Emerg. Manage., vol. 2, pp. 26-28, 2020.

[10] L. M. Suo, "Analysis on the coordination of vertical intergovernmental relations in cross-regional environmental governance," Study Local Gov., vol. 1, pp. 57-69, 2019.

[11] A. S. J. Goetz and D. L. Debrtin, "The environmental Kuznets Curve for U. S. Counties: A spatial econometric analysis with extensions," Pap. Reg. Sci., vol. 2, pp. 407-424, 2010.

[12] F. H. Chai, Y. R. Yun, and S. L. Wang, "Study on implementation of joint prevention and control of regional air pollution mechanism," Environ. Sustain. Dev., vol. 4, pp. 5-9, 2013.

[13] Y. Shu, H. C. Wang, J. N. Hu, and Y. M. Sun, "Study on the long-term and effective mechanism of regional air pollution joint prevention and control," Environ. Sustain. Dev., vol. 4, pp. 78-81, 2019.

[14] J. Zheng, "Inspiration for China from the long-term mechanism of trans-regional cooperation in air pollution prevention and control in Europe," Environ. Protect., vol. 45, no. 5, pp. 75-77, 2017.

[15] H. Yang and J. P. Liu, "Research on spatio-temporal diffusion of regional air pollution and its joint prevention and control strategy," Eng. Manage. Technol., vol. 6, pp. 196-197, 2018.

[16] P. C. Shi, H. M. Wang, S. Z. Yang, C. Chen, and W. T. Yang, "Blockchain-based trusted data sharing among trusted stakeholders in IoT," Softw. Pract. Exp., vol. 5, pp. 1028-1040, 2019.
[17] J. W. Zhang, W. X. Cui, J. F. Ma, and C. Yang, "Blockchain-based secure and fair crowd sourcing scheme," Int. J. Distrib. Sens. Networks, vol. 15, no. 7, pp. 78-90, 2019.

[18] L. Cai, "Blockchain can provide technical support for data sharing during epidemic prevention," [EB/OL], https://www.qklw.com/lives/ 20200224/56723.html, 2020.

[19] Q. Liu and X. Zou, "Research on trust mechanism of cooperation innovation with big data processing based on blockchain," EURASIP J. Wireless Commun. Netw., vol. 26, pp. 1-11, 2019.

[20] H. W. Zhao, W. Cui, S. W Li, and R. Z. Xu, "Token economy: A new form economy with decentralized mutual trust and collective governance," in IEEE 14th Int. Symp. Auton. Decentralized Syst. (ISADS), Dutch, Mar. 8-10 2019, pp. 113-119.

[21] D. Yang and C. H. Yu, "The application of block chain technology in government governance and social governance," Governance, vol. 11, pp. 43-48, 2019, doi: 10.16619/j.cnki.cn10-1264/d.2019.43.006.

[22] Q. Q. Pei, D. L. Ma, and L. P. Zhang, "Block chain and digital reconstruction of social governance," J. Xinjiang Normal Univ., vol. 5, pp. 12-20, May 2020.

[23] N. Z. Aitzhan and D. Svetinovic, "Security and privacy in decentralized energy trading through multi-signatures, blockchain and anonymous messaging stream," IEEE. Trans. Dependable Secure Comput., vol. 3, no. 5, pp. 840-852, 2018.

[24] Y. Chen and H. Hu, "Internet of intelligent things and robot as a service," Simul. Model. Pract. Theory, vol. 34, pp. 159-171, May 2013.

[25] Y. Chen, Service-oriented Computing and System Integration: Software, IoT, Big Data, and AI as Services, 7th ed., Kendall Hunt Publishing, Dubuque, 2020. 